\title{
$50 S$ subunit recognition and modification by the Mycobacterium tuberculosis ribosomal RNA methyltransferase TlyA
}

Zane T. Laughlin ${ }^{1,2}$, Debayan Dey ${ }^{1}$, Natalia Zelinskaya ${ }^{1}$, Marta A. Witek ${ }^{1}$, Pooja Srinivas ${ }^{1,3}$, Ha An Nguyen $^{1,4}$, Emily G. Kuiper ${ }^{1}$, Lindsay R. Comstock ${ }^{5}$, Christine M. Dunham ${ }^{1,6}$ and Graeme L. Conn ${ }^{1,6 *}$

${ }^{1}$ Department of Biochemistry, Emory University School of Medicine, Atlanta, GA, 30322, USA.

${ }^{2}$ Graduate Program in Biochemistry, Cell and Developmental Biology (BCDB), Graduate Division of Biological and Biomedical Sciences, Emory University, Atlanta, GA, 30322,USA.

${ }^{3}$ Graduate Program in Molecular and Systems Pharmacology, Graduate Division of Biological and Biomedical Sciences, Emory University, Atlanta, GA, 30322,USA.

${ }^{4}$ Department of Chemistry Graduate Program, Emory University, Atlanta, GA, 30322,USA

${ }^{5}$ Department of Chemistry, Wake Forest University, 455 Vine Street, Wake Downtown, NC, 27101, USA.

${ }^{6}$ Emory Antibiotic Resistance Center (ARC), Emory University, Atlanta, 30322, GA, USA.

*To whom correspondence should be addressed, email: gconn@emory.edu.

Competing Interest Statement: The authors declare no competing interests.

Running title: Structure of the 50S-TlyA complex

Key Words: Ribosome, RNA modification, antibiotic resistance, mycobacteria, methyltransferase. 


\begin{abstract}
Changes in bacterial ribosomal RNA (rRNA) methylation status can alter the activity of diverse groups of ribosome-targeting antibiotics. Typically, such modifications are incorporated by a single methyltransferase that acts on one nucleotide target and rRNA methylation directly prevents drug binding, thereby conferring drug resistance. However, loss of intrinsic methylation can also result in antibiotic resistance. For example, Mycobacterium tuberculosis (Mtb) becomes sensitized to tuberactinomycin antibiotics, such as capreomycin and viomycin, due to the action of the intrinsic methyltransferase TlyA. TlyA is unique among antibiotic resistance-associated methyltransferases as it has dual $16 \mathrm{~S}$ and $23 \mathrm{~S}$ rRNA substrate specificity and can incorporate cytidine-2'-O-methylations within two structurally distinct contexts. How TlyA accomplishes this feat of dual-target molecular recognition is currently unknown. Here, we report the structure of the Mtb 50S-TlyA subunit complex trapped in a post-catalytic state with a S-adenosyl-L-methionine analog using single-particle cryogenic electron microscopy. This structure, together with complementary site-directed mutagenesis and methyltransferase functional analyses, reveals critical roles in 23S rRNA substrate recognition for conserved residues across an interaction surface that spans both TlyA domains. These interactions position the TlyA active site over the target nucleotide C2144 which is flipped from $23 \mathrm{~S}$ Helix 69 in a process stabilized by stacking of TlyA residue Phe157 on the adjacent A2143. This work reveals critical aspects of substrate recognition by TlyA and suggests that base flipping is likely a common strategy among rRNA methyltransferase enzymes even in cases where the target site is accessible without such structural reorganization.
\end{abstract}

\title{
Introduction
}

Ribosome-targeting antibiotics are a structurally and mechanistically diverse group of anti-infectives that comprise a significant proportion of currently used treatments for bacterial infections $(1,2)$.

However, among resistance mechanisms exploited by pathogenic bacteria to evade the effects of these antibiotics, ribosomal RNA (rRNA) drug-binding site methylation is already established or is quickly emerging as a major threat to the efficacy of such treatments. For example, resistance modifications impacting the efficacy of macrolides $(3,4)$ and aminoglycosides $(5,6)$ that are incorporated by Sadenosyl-L-methionine (SAM)-dependent methyltransferases have been acquired by diverse human pathogens. Less commonly, loss of intrinsic methylation can also lead to resistance, such as streptomycin resistance through loss of the methyltransferase RsmG/GidB $(7,8)$, and capreomycin resistance in Mycobacterium tuberculosis (Mtb) through loss of TlyA (9).

Capreomycin is a member of the tuberactinomycin class of ribosome-targeting antibiotics and has an important history in the treatment of $M t b$ infections resistant to the first-line drugs rifampin and isoniazid (10). Capreomycin binds at the subunit interface of mature $70 S$ ribosomes, adjacent to $16 \mathrm{~S}$ 
rRNA helix 44 (h44) of the small (30S) subunit and 23S rRNA Helix 69 (H69) of the large (50S) subunit (11). In a recent study, the tuberactinomycin antibiotic viomycin was also found to bind the $70 S$ ribosome at several other locations (12), suggesting that this class of antibiotics may target multiple ribosomal sites to interfere with translation. Capreomycin's activity is thought to arise via stabilization of tRNA in the A site of the ribosome, thereby halting translation (11). Capreomycin has also been proposed to disrupt the interaction of ribosomal proteins $L 10$ and $L 12$, thereby blocking binding of elongation factors during translation (13). However, this mechanism is harder to reconcile with the binding sites of capreomycin and viomycin which are distant from both $L 10$ or $L 12(11,12,14)$, as well as the impact of changes in rRNA modification status in the A site on their activity.

Capreomycin binding to the Mtb 70S ribosome is dependent on 2'-O methylation of two nucleotides at the subunit interface, 16S rRNA C1392 and 23S rRNA C2144 (corresponding to E. coli nucleotides C1409 and C1920, respectively) (15). While the precise role of these modifications in ribosome structure and function is currently unclear, it is thought that they may somehow change the conformation of the rRNA allowing for optimal capreomycin binding $(15,16)$. Evolutionary maintenance of intrinsic rRNA modifications which increase sensitivity to antibiotics may be driven by their contribution to optimal fitness in the absence of drug or through decreased stability of unmodified 70S, as observed in Mycolicibacterium smegmatis (Msm) and Campylobacter jejuni, respectively (17, 18). Both modifications are incorporated by a single SAM-dependent ribose 2'-O-methyltransferase, TlyA (encoded by Rv1694 in Mtb) (16), which acts on the individual mature ribosomal subunits. The TlyA family of methyltransferases is also divided into two groups based on their substrate specificities: Type I TlyA (TlyA') exclusively methylate 23S rRNA, while the slightly larger TlyA", including the Mtb enzyme, possess dual $16 \mathrm{~S}$ and $23 \mathrm{~S}$ specificity (15). However, how Mtb TlyA and other TlyA" enzymes recognize and modify these two structurally distinct substrates is not currently known.

We previously determined the crystal structure of the C-terminal domain (CTD) of Mtb TlyA with and without a four amino acid interdomain linker sequence (19). The TlyA CTD adopts the expected Class I methyltransferase fold but was unexpectedly found to be deficient in SAM binding in the absence of the interdomain linker. A TlyA CTD structure including the linker also revealed that this short motif can either extend the first $\alpha$-helix of the CTD, or form a loop structure similar to that proposed earlier via homology modeling $(19,20)$. While a structure of the TlyA N-terminal domain is currently not available, modeling suggests a ribosomal protein S4-like domain (20). Collectively, these findings suggest that the N-terminal domain may be essential for rRNA recognition and binding, with the interdomain linker potentially playing a role in promoting SAM binding and methyltransferase activity in the CTD once bound to the correct substrate (19).

Here, we describe the structure of full-length Mtb TlyA bound to the Msm 50 S subunit. The structure reveals the critical role played by the TlyA NTD in recognizing a complex 23S rRNA structure at the base of $\mathrm{H69}$, positioning the TlyA CTD on $\mathrm{H} 69$ with its active site over the target nucleotide, 
C2144 (in Msm numbering, which is used exclusively hereafter unless noted). In addition, we find that TlyA uses a mechanism of base-flipping for target site recognition and modification, despite the accessibility of the $\mathrm{C} 2144$ ribose 2'-OH in $\mathrm{H} 69$ suggesting this may be a general strategy for substrate molecular recognition among rRNA methyltransferases.

\section{Results}

Determination of the 50S-TIyA complex structure-50S ribosome subunits without ribose modification on 23S rRNA nucleotide C2144 were isolated from Msm strain LR222 C101A which lacks TlyA activity. Mtb TlyA was expressed in E. coli and purified as previously described (19) and a SAManalog, "N-mustard 6" (NM6), used to increase occupancy of TlyA on the $50 \mathrm{~S}(21,22)$. This is accomplished by the transfer of NM6 by TlyA in its entirety to the ribose 2'-OH of C2144 and its covalent attachment to the 23S rRNA thus stabilizes the 50S-TlyA complex by virtue of the enzyme's affinity for both substrate and SAM analog (Fig. S1). Using this approach, we determined a $3.05 \AA$ resolution overall map of TlyA bound to the $50 \mathrm{~S}$ in a state immediately after catalysis of C2144 ribose modification by single-particle cryogenic electron microscopy (cryo-EM) (Fig. 1, Fig. S2, Fig. S3A-C).

One 23S rRNA feature, H54a (also called the "handle"), was significantly shifted from its position in the previously solved Msm 70S structure (PDB code 5060) (23) where it makes extensive interactions with the $30 \mathrm{~S}$ subunit. This feature was visible in some 3D reconstructions (Fig. S4), where $\mathrm{H} 54 \mathrm{a}$ lies across the subunit interface surface of the 50S. In contrast, in other reconstructions, the map quality was very weak, suggesting $\mathrm{H} 54 \mathrm{a}$ is dynamic in the free $50 \mathrm{~S}$. H54a's variable position and weak map adjacent to the bound TlyA in most 3D reconstructions suggest that H54a does not contribute to TlyA interaction with 50 S despite its proximity to the enzyme NTD (Fig. S4).

An initial model for the 50S-TlyA complex was produced by docking a model of full-length TlyA, previously produced using a combination of NTD homology model and CTD crystal structure (PDB code 5KYG) (19), into unoccupied density surrounding 23S rRNA H69. This TlyA structure was subsequently rebuilt in Coot (24), including a complete rebuilding of the NTD (see Methods and Materials for details). Although the map was of sufficient quality for initial rebuilding of the NTD, the region corresponding to the TlyA CTD was less well defined. We therefore also performed multibody refinement with $\mathrm{H} 69$ and TlyA masked to separate this specific region of interest from the remainder of the 50 S (Fig. S2). This multibody refinement produced separate maps of the H69:TlyA complex (3.61 $\AA$ following post-processing; Figs. S3D-F) and the remaining 50 S lacking $\mathrm{H} 69$ (2.99 $\AA$ following postprocessing; Figs. S3G-I). The former map was significantly improved compared to the corresponding region of the original map, with more information on the secondary structure and side chains of TlyA providing insights into how full-length TlyA interacts with its $23 S$ rRNA substrate. Each map from multibody refinement was used for final model building and refinement of its associated structure, and 
the separate structures combined to generate a complete model of the 50 S subunit-TlyA complex (Fig. 2, Fig. S2).

When bound to the 50S subunit, the TlyA CTD is essentially identical to the previously determined structure of the isolated domain (PDB code 5KYG; $2.45 \AA$ RMSD for 209 CTD C $\alpha$ atoms), with the exception of a significant movement ( $\sim 6-8 \AA)$ of the loop containing residues 114-117 that is necessary to avoid clash with the minor groove surface of H69 (Fig. S5A,B). TlyA is structurally similar to the putative $S$. thermophilus hemolysin (PDB code 3HP7), but with a significant difference in the relative NTD and CTD orientation as a result of the distinct backbone path at the linker between the domains (Fig. S5C,D). The structures thus align well for superpositions based on either individual domain (3.68 and 2.00 $\AA$ RMSD for 209 CTD or 59 NTD $C \alpha$ atoms, respectively), but less well for the full protein (overall 7.13 $\AA$ RMSD for $268 \mathrm{C} \alpha$ atoms). The final NTD model reveals a globular domain with expected similarity to ribosomal protein S4 (2.55 A RMSD for 59 NTD C $\alpha$ atoms; Fig. S5E), comprising two adjacent short $\alpha$-helices (residues 6-14 and 20-28) and two short $\beta$-strands (residues 32-24 and 52-54) preceding and interdomain linker (residues 60-63).

As described further in the following sections, TlyA binds $50 \mathrm{~S}$ on its subunit interface surface, with both TlyA domains surrounding $\mathrm{H} 69$ and the NTD making additional contacts to the rRNA junction at the base of the H69 (Fig. 2A-D). Together, the two TlyA domains form a continuous largely positively charged surface in contact with the 23 rRNA, suggesting that both play an important role in $50 \mathrm{~S}$ binding and specific substrate recognition (Fig. 2D,E). The final model also reveals the CTD of TlyA with bound SAM analog NM6 positioned directly over H69 residue C2144 in a post-catalytic state (i.e., with C2144 modified with NM6) .

\section{TlyA NTD residues Arg6 and Arg20 exploit non-canonical rRNA structure for specific substrate} recognition-Eight residues in the TlyA NTD were identified to make potentially critical interactions with nucleotides at the base of $\mathrm{H} 69$ and the adjacent rRNA junction: Arg4, Arg6, Arg18, Ser19, Arg20, Gln21, Gln22, and Lys41 (Fig. 3A). Three of these residues, Arg4, Arg6 and Arg20, are clustered around the non-canonical RNA structure formed by residues C2149-G2153 of the 23 rRNA sequence immediately following H69 (Fig. 3B). While Arg4 is positioned to form a single electrostatic interaction with the phosphate group of A2151, Arg6 and Arg20 each form interaction networks with multiple rRNA nucleotides and with each other, likely stabilized by additional interactions with Asp8 (Fig. 3B). Specifically, Arg6 recognizes a sharp turn in the rRNA backbone via contacts with the bridging oxygen of A2151 and non-bridging oxygens of A1552, as well as a cation- $\pi$ stacking interaction on the nucleobase of U2150. Similarly, Arg20 recognizes the phosphate backbone of 23S rRNA via electrostatic interactions with the phosphate groups of C2149 and U2150 (Fig. 3B). Consistent with critical roles in 23S rRNA recognition for Arg6 and Arg20, these two residues, as well as Asp8, are almost universally conserved among TlyA homologs (Fig. 3C, Fig. S6). 
To confirm the importance of Arg6 and Arg20 residues in 23S rRNA recognition, individual alanine substitution variants were created and their proper folding was confirmed using nano-differential scanning fluorimetry (nDSF; Fig. S7). Next, enzyme activity was assessed in two complementary methyltransferase activity assays: quantification of $50 \mathrm{~S}^{3} \mathrm{H}$ incorporation following transfer of a $\left[{ }^{3} \mathrm{H}\right]-$ methyl group from radiolabeled SAM cosubstrate ([ $\left.\left.{ }^{3} \mathrm{H}\right]-\mathrm{SAM}\right)$ and direct visualization of C2144 2'-Omethylation via reverse transcription (RT) primer extension. For the $\left[{ }^{3} \mathrm{H}\right]-\mathrm{SAM}$ assay, we first established optimal conditions using wild-type TlyA and then compared these and all other variants in a single time-point assay under conditions corresponding to $\sim 90 \%$ completion of $50 \mathrm{~S}$ methylation for the wild-type enzyme (Fig. S8). Consistent with an essential role in specific 50 S substrate recognition, individual substitution of either Arg6 or Arg20 completely eliminated methyltransferase activity (Fig. 3D). This result was corroborated in the RT assay in which no methylation above background at $\mathrm{C} 2144$ was observed for either TlyA R6A or R20A (Fig. 3E). In contrast, TlyA R4A exhibited some activity in the $\left[{ }^{3} \mathrm{H}\right]-S A M$ assay and more robust methylation via primer extension, suggesting this residue makes a smaller contribution to $50 \mathrm{~S}$ binding by TlyA, as previously noted (15). While the reason for difference between the two assays in R4A variant activity is not immediately obvious, the RT assay is less readily amenable to accurately assessing complete methylation for wild-type TlyA and thus for quantitative comparison with variants.

Four other residues surrounding Arg20 are also positioned to make interactions with $\mathrm{H} 69$ nucleotides A2146, U2147 and C2148, as well as the TlyA-bound SAM analog. Arg18 is adjacent to one non-bridging oxygen of the phosphate group of U2147, while Gln21 is located between the second non-bridging oxygen of the same phosphate group, the base $\mathrm{O} 4$ atom of U2147, and the terminal carboxyl group of the bound SAM analog (Fig. 3F). Ser19 is also located between these residues and the phosphate of $\mathrm{C} 2148$, with $\mathrm{Gln} 22$ in a central location within 3-4 $\AA$ of all three other TlyA residues as well as the U2147 phosphate group. As before, these residues were substituted with alanine (R18A and $\mathrm{S} 19 \mathrm{~A}$ ) or as a double change with a more conservative asparagine substitution at both glutamine residues (Q21N/Q22N) and assessed in the two activity assays after confirming their correct folding (Fig. 3D,E, Fig. S7B,D). Consistent with their more modest conservation among TlyA homologs compared to Arg6 and Arg20, all three variant proteins were affected by the amino acid substitution but retained some activity in both assays (Fig. 3C-E, Fig. S6). These results suggest these residues play supporting, but not individually critical, roles in TlyA substrate recognition

Finally, within the TlyA NTD, the highly conserved Lys41 was observed to make a single electrostatic interaction with the phosphate group of $\mathrm{C} 2055$ which is located in a bulge loop at the base of $\mathrm{H68}$, on the strand complementary to the 23S rRNA sequence preceding H69 (Fig. 3C,G, Fig. S6). Again, a reduction in both activity assays was observed suggesting an important, but not individually critical, role in $50 S$ binding for Lys41 (Fig. 3D,E). Thus, these analyses have identified the NTD residues that contribute collectively to 50 S interaction (Arg4, Arg18, Ser19, Gln21, GIn22, and Lys41), 
including two, Arg6 and Arg20, whose coordinated recognition of a non-canonical 23S rRNA structure adjacent to $\mathrm{H} 69$ is critical for specific $50 \mathrm{~S}$ substrate recognition by TlyA.

\section{TlyA CTD interactions with $\mathrm{H69}$ position the methyltransferase domain for C2144 modification-}

The TlyA CTD makes extensive contact with the irregular minor groove of H69, from the U2132:A2146 pair near the base to the tip of the helix, with two positive patches on either side of the SAM binding pocket extending the NTD contact surface on the rRNA (Fig. 2). Five TlyA residues of moderate to very high conservation are positioned to interact with H69: Arg65, Tyr115, Arg133, Arg137, and Lys189 (Fig. 4A-D). Two additional residues at the C2144 target nucleotide, Phe157 and Ser234, and their role in TlyA activity are described further in the next section. As before, each residue was individually substituted with alanine, and additionally to isoleucine in the case of Tyr115 to specifically probe the requirement for an aromatic side chain at this position. The purified variant proteins were assessed using nDSF (Fig. S7) which revealed them to be properly folded with only one potential exception, Y115I, which retained an unfolding temperature $\left(T_{i}\right)$ similar to the wild-type protein but with an inverted profile (Fig. S7E).

Towards the base of $\mathrm{H69}$, Arg65 recognizes the phosphate backbone of nucleotides A2146 and U2147 with the guanidine head group positioned beneath the phosphate of A2146 and within electrostatic interaction distance of a non-bridging oxygen of the U2147 phosphate (Fig. 4B). On the opposite stand of H69, Tyr115 extends into the minor groove, contacting the G2134 ribose and G2133 ribose and base edge, with the hydroxyl group within hydrogen bonding distance of the G2133 nucleobase N3 atom (Fig. 4B). Positioning of Tyr115 to make these interactions also depends upon a local but significant loop reorganization between the free and 50S-bound forms of TlyA (Fig. S5B). Arg65 is universally conserved and substitution with alanine completely ablates activity in both assays (Fig. 4E-G, Fig. S6), consistent with a critical role in $50 \mathrm{~S}$ binding and substrate recognition. Substitution of Tyr115 with either alanine or isoleucine similarly results in fully diminished enzyme activity in both assays (Fig. 4F-G). Although Tyr115 is not as highly conserved as Arg65, this position is most commonly aromatic and/ or basic (e.g. tyrosine, histidine or arginine; Fig. 4E, Fig. S6), suggesting conservation of interactions like those we observe in the structure is essential in other TlyA homologs.

Three basic TlyA residues (Arg133, Arg137, and Lys189) surround the hairpin loop structure at the tip of ribosomal H69. Arg133 and Arg137 approach the backbone phosphate groups on the minor groove side of nucleotides U2135/A2136 and A2138, respectively, while on the opposite side of H69, Lys189 is positioned alongside the base edges of A2136 and U2141 (Fig. 4C,D). Arg137 is highly conserved among TlyA homologs (85-90\%), whereas conservation is more modest at the other two positions, though still most commonly a basic residue ( 40-77\% Arg/Lys; Fig. 4E, Fig S6). Functional analyses of individual alanine substitution variants at these residues revealed a modest impact on TlyA activity with all three comparably reduced in the $\left[3^{\mathrm{H}}\right]$-SAM assay, but only R137A exhibiting significantly 
diminished activity in the RT assay (Fig. 4F,G). While these results suggest that Arg133, Arg137, and Lys 189 contribute to $\mathrm{H} 69$ binding by TlyA, this may be accomplished through their collective interactions with the tip of the helix.

Together, our structural insights and functional analyses suggest that the TlyA CTD contains at least two residues critical for $\mathrm{H} 69$ binding, Arg65 and Tyr115, and several others that collectively recognize features along the length of $\mathrm{H69}$. Further, these residues lie on a contiguous surface with similarly essential NTD residues (Arg6 and Arg20), suggesting coordinated recognition of distinct features of $23 S$ rRNA underpin specific substrate recognition of the $50 \mathrm{~S}$ subunit by TlyA.

TlyA catalytic mechanism-Docking of TlyA on the 50S precisely positions the opening to the SAM binding pocket and TlyA active site directly over the target nucleotide C2144 (Fig 5A). As noted earlier, use of NM6 in preparing the 50S-TlyA complex also facilitated capture of the enzyme in a post-catalytic state, with C2144 covalently modified on its 2'-OH and the SAM analog still bound in TlyA's cosubstrate binding pocket. While much of $\mathrm{H} 69$ and the adjacent rRNA junction is structurally unaltered upon TlyA binding suggesting that the enzyme specifically recognizes the mature $50 S$ subunit, we observe significant local deformations around the target nucleotide in our structure. Most strikingly, C2144 is fully flipped out of the $\mathrm{H} 69$ base stacking, with two TlyA residues, Phe157 and Ser234, positioned to stabilize the altered H69 structure (Fig. 5B-D). Phe157, which is almost universally conserved among TlyA homologs (Fig. 5C, Fig. S6), stacks on A2143 and partly fills the space normally occupied by C2144. This interaction appears mechanistically critical as substitution of the side chain completely abrogates activity (Fig. 5D-F). Further, both F157A and F157I variants are inactive suggesting that the $\pi-\pi$ stacking of aromatic side chain and nucleobase is essential. In contrast, the observed interaction of Ser234 via its hydroxyl group with the $\mathrm{NH}_{2}$ of $\mathrm{C} 2144$ does not appear essential for activity, consistent with the very low level of conservation at this position (Fig. S6). As such, despite being suggested by the structure, TlyA does not require direct identification of the base identity at C2144 for modification (Fig. 5D-F). However, we also note that Ser234 is flanked by two universally conserved glycine residues which likely impart the necessary flexibility in this short loop to intimately sequester the flipped base which may allow some level of discrimination among possible RNA bases.

\section{Discussion}

Changes in rRNA modification status can have profound effects on ribosome assembly, function, and sensitivity to ribosome-targeting antibiotics (25). rRNA methylations have been identified which either block antibiotic action or are necessary for optimal drug binding and thus antibacterial activity. In bacteria, these rRNA methylations are incorporated by Class I or Class IV methyltransferases (26, 27), with a single enzyme typically responsible for each individual modification. Exceptions to this strict specificity do exist, most strikingly for TlyA which is capable of incorporating cytidine 2'-O-methyl 
modifications on both the small and large subunit, within two structurally distinct contexts.

Here, we determined the cryo-EM structure of TlyA bound to the $50 \mathrm{~S}$ ribosomal subunit, revealing the full-length structure of the enzyme and the molecular mechanism of specific recognition of one of its two ribosomal subunit substrates. To our knowledge, this structure represents the first example of an rRNA 2'-O methyltransferase bound to the bacterial $50 \mathrm{~S}$ ribosomal subunit. These studies also identified an essential 23S rRNA interaction surface that spans both the NTD and CTD of TlyA and contains a set of residues critical for 50 S substrate binding and 2'-O-methylation of C2144. In particular, TlyA accomplishes specific $50 \mathrm{~S}$ recognition via essential interactions of its NTD with a unique tertiary structure at the base of $\mathrm{H} 69$ and of the CTD with $\mathrm{H} 69$, which position the bound methyl group donor SAM over the target nucleotide. Finally, the nucleobase of C2144 is flipped out of the H69 helical stack, in a conformation stabilized by TlyA Phe157 stacking on the adjacent A2143, placing the 2'-OH of the ribose of C2144 adjacent to SAM and the catalytically important residues of TlyA (20).

The TlyA NTD adopts an S4 ribosomal protein fold that makes critical interactions, primarily by the highly conserved TlyA residues Arg6 and Arg20, with the non-canonical 23S rRNA structure of the junction at the base of H69. In the original report of the S4 structure from Bacillus stearothermophilus, the corresponding S4 residues (Arg92 and Arg106 in the Msm S4 domain 2; Fig. S5F,G) were among several highly conserved basic or aromatic residues proposed to form an extensive rRNA binding surface (28). Many of these predictions were confirmed by subsequent bacterial ribosome structures showing the S4 and TlyA NTD interaction surfaces with rRNA have significant overlap including Arg104 (TlyA Arg18) and through non-conserved residues corresponding to TlyA Arg4 and Lys41. Critically, however, S4 Arg92 and Arg106 do not make extensive interactions with 16S rRNA, with Arg92 positioned closest ( 4.6 $\AA$ ) and Arg106 directed away from the rRNA in the Msm 70S structure (23). Instead, these two residues appear to play important roles in S4 interdomain interactions, with Tyr60 from S4 domain I sandwiched between them and forming a cation- $\pi$ stacking interaction with Arg106. Further distinguishing the organization of these residues in TlyA and S4, Arg92 and Arg106 are $7 \AA$ apart and do not interact, and only Arg92 makes interactions with Asp94 (the equivalent of the conserved Asp8 in TlyA). Thus, in TlyA, Arg6 and Arg20 have been repurposed compared to S4 to directly recognize the $23 \mathrm{~S}$ rRNA structure, in a manner supported by Asp8 that is also unique to TlyA.

Our structure revealed $\mathrm{C} 2144$ to be flipped out of $\mathrm{H} 69$ in a post-catalytic state captured by use of the SAM analog. This observation was somewhat unexpected based on the relative accessibility of the $\mathrm{C} 2144$ ribose 2'-OH in the $\mathrm{H} 69$ minor groove, but such base flipping has been observed or proposed for other rRNA methyltransferases and may thus be a common mechanistic feature of such enzymes. Comparisons to the well characterized $16 \mathrm{~S}$ rRNA ( $\mathrm{m}^{1} \mathrm{~A} 1408$; E. coli numbering) aminoglycoside-resistance Class I methyltransferases, such as NpmA (29), are particularly intriguing. NpmA also uses a base-flipping mechanism despite the $\mathrm{N} 1$ atom being relatively exposed on the helix 44 surface. Like TlyA, NpmA relies heavily on recognition of complex rRNA structure, distant from the 
site of modification, to accomplish specific binding to its substrate (29). Further, a single direct base edge contact is made by NpmA to A1408, but the absolute importance of this interaction is unclear given that NpmA retains partial activity against ribosomes with a G1408 nucleotide (30). Similarly, TlyA contacts the nucleobase of C2144 via Ser234 located in the loop linking the sixth and seventh $\beta$ strands ( $\beta 6 / 7$ linker) of its Class I methyltransferase core fold, a region commonly associated with substrate recognition by these enzymes $(27,31)$. As for NpmA, this specific base contact does not appear critical for TlyA activity based on our functional analyses. However, as noted earlier, the high conservation of the surrounding sequence suggests the TlyA $\beta 6 / 7$ linker structure may nonetheless be important for forming the pocket shielding C2144 from exposure to solvent in its flipped conformation. One distinction between TlyA and NpmA appears to be how the flipped conformation is stabilized. In NpmA, a basic residue (Arg207) stabilizes a local distortion of the h44 backbone, and the flipped A1408 is stabilized by stacking between two conserved tryptophan residues while the vacated space within helix 44 is left unoccupied. In contrast, TlyA uses the conserved Phe157 to occupy the space vacated by flipping of $\mathrm{C} 2144$ via stacking on the adjacent A2143 nucleobase. As such, the mechanism used by TlyA is more akin to DNA methyltransferases which replace DNA-DNA interactions (base pairing and stacking) with protein-DNA contacts within the helix (32).

The similarities and distinctions between TlyA and NpmA may also be significant for TlyA's mechanism of recognition of its other target nucleotide in the 30S, 16S rRNA nucleotide C1392 (C1409 in E. coli), immediately following A1408 in the 16S rRNA. Our speculation is that TlyA may exploit the same complex $16 \mathrm{~S}$ rRNA tertiary surface used by NpmA and related enzymes, as well as the $\mathrm{m}^{7} \mathrm{G} 1405$ aminoglycoside-resistance methyltransferases (33). Whether the same surfaces of TlyA are engaged in recognition of $30 \mathrm{~S}$ and with the same strict dependencies on specific key residues for rRNA interaction (e.g. Arg6, Arg20 and Arg65) and stabilization of base flipping by Phe157 are currently not known. We have also previously proposed that structural plasticity in the short interdomain linker in TlyA may be a mechanism by which the enzyme can adapt its structure to the two distinct interaction surfaces presented on the $50 \mathrm{~S}$ and $30 \mathrm{~S}$ (19). In further support of this idea, a known clinical capreomycin resistance mutation resulting in an A67E substitution (34) would likely disrupt the hydrophobic binding pocket which linker residue Trp62 occupies in the 50S-bound TlyA structure, which could thus prevent SAM binding or correct NTD/ CTD association or interdomain communication.

Additional insight can also be gleaned from the existence of two TlyA subtypes, TlyA' and TlyA", of which only the longer TlyA" possesses dual substrate specificity and is able to modify $30 \mathrm{~S}$. The key elements of $50 S$ recognition identified here are conserved between the two enzyme subgroups, but TlyA' enzymes lack a short sequence at their $\mathrm{N}$-terminus and an entire $\alpha$-helix that follows the seventh core $\beta$-strand in TlyA". Thus, potentially critical elements of 305 recognition may reside in these regions. In support of this, previous studies with TlyA N-terminal truncations suggest a functional role for residues Arg3 and/ or Arg4 (15). However, fully defining the basis of TlyA's dual substrate specificity 
will require corresponding detailed structure-function studies of TlyA and its 305 substrate.

Of the two modifications incorporated by TlyA, C2144 methylation most strongly influences the binding of capreomycin (approximately $20 \AA$ away) by a long-range mechanism that is not currently well defined. Comparison of $\mathrm{H} 69$ in multiple ribosome structures available in the PDB with and without C2144 modification reveals a small but consistent difference at the tip H69: in unmodified ribosomes, the loop formed by nucleotides A2137, C2138, and U2139 makes a tighter turn than in modified ribosomes. Our structure now offers a third comparison, with a bulkier modification incorporated but with TlyA still also bound, in which $\mathrm{H69}$ is observed in an structural state between those of ribosomes with unmodified and modified C2144. In the conformation of other unmodified bacterial ribosomes, when $\mathrm{H} 69$ is more tightly bent, the bases of A2137 and C2138 are more distant from the capreomycin binding site on the $30 \mathrm{~S}$. These observations suggest that modification of C2144 alters the structure of H69 in a manner that changes the position of nucleotides A2137 and C2138, promoting the direct interactions they make with capreomycin.

Clinical resistance to capreomycin can arise through $16 \mathrm{~S}$ rRNA mutation $(34,35)$ or, as noted above, via amino acid substitutions in TlyA that eliminate its activity and thus incorporation of the rRNA methylation required for optimal capreomycin activity $(34,36-38)$. Our structure also offers further insight into the latter mechanism of clinical resistance. In the TlyA NTD, a mutation was identified that results in an R14W substitution (34) which likely disrupts TlyA NTD folding and its essential contribution to $50 \mathrm{~S}$ substrate recognition. Although it does not directly contact $23 \mathrm{~S}$ rRNA, Arg14 is positioned directly above Arg6 and Arg20, and interacts with the TlyA backbone at Thr50/Ala51 which are part of a loop that wraps closely around the arginine side chain. Thus, structural changes to accommodate the bulkier tryptophan side chain would disrupt the critical interactions with the rRNA made by Arg6 and Arg20. Another common mutation found in resistant Mtb results is a N236K substitution (37) which our structure suggests could impact TlyA activity in several ways. Gln236 immediately follows the $\beta 6 / 7$ linker (sequence ${ }^{232} \mathrm{GPSG}^{235}$ ) which surrounds the flipped C2144 base. Additionally, this substitution places a lysine residue close to residue Glu238 which has been proposed to play an important role in catalysis (20).

In summary, the present work has revealed the full-length structure of the Mtb methyltransferase TlyA and defined the molecular basis for specific recognition of its $50 \mathrm{~S}$ subunit substrate. While future structural and biochemical studies with the $30 \mathrm{~S}$ subunit will be necessary for a full understanding of TlyA's dual substrate specificity, these studies have deepened our understanding of rRNA methyltransferase action. In particular, recognition of unusual rRNA structures distant from the site of modification and base flipping both emerge as general themes in substrate molecular recognition for these enzymes.

\section{Materials and Methods}


TlyA protein expression, purification and site-directed mutagenesis-An E. coli codon-optimized sequence encoding Mtb (strain ATCC 25618/H37Rv) TlyA was obtained via chemical synthesis (GeneArt) and subcloned into a pET44a(+) plasmid (pET44-TlyA), as previously described (19). This construct produces TlyA with an N-terminal hexahistidine tag. The TlyA-encoding plasmid was used to transform E. coli BL21 (DE3) and cultures were grown at $37^{\circ} \mathrm{C}$ in Terrific Broth containing $100 \mu \mathrm{g} / \mathrm{mL}$ ampicillin. At mid-log phase ( 0.4-0.6 OD 600$)$ protein expression was induced with $0.5 \mathrm{mM}$ isopropyl $\beta$ D-1-thiogalactopyranoside, and growth continued for an additional 3.5 hours. Following harvest via low speed centrifugation for 10 minutes, the cells were resuspended in lysis buffer $\left(50 \mathrm{mM} \mathrm{NaH}_{2} \mathrm{PO}_{4}, \mathrm{pH}\right.$ 8.0, $300 \mathrm{mM} \mathrm{NaCl}$, and $10 \mathrm{mM}$ imidazole containing an EDTA-free SIGMAFAST ${ }^{\mathrm{TM}}$ Protease Inhibitor Cocktail Tablet) and lysed by sonication (Misonix Sonicator 3000 with microtip: 15 minutes total sonication time, $0.9 \mathrm{~s}$ on, $0.6 \mathrm{~s}$ off, output level 5.5$)$. Cell lysates were cleared by centrifugation $(21,000$ $\mathrm{x} g$ ) for 40 minutes and filtered before purification of TlyA by sequential $\mathrm{Ni}^{2+}$-affinity (Cytiva HisTrap ${ }^{\mathrm{TM}}$ FF crude $1 \mathrm{~mL}$ or manual His-column using Millipore Ni-NTA His-Bind $囚$ Resin) and gel filtration (Cytiva HiLoad $^{\mathrm{TM}}$ 16/600 Superdex ${ }^{\mathrm{TM}}$ 75) chromatographies on an ÄKTA Purifier 10 system. TlyA variants with single or double amino acid substitutions were created using megaprimer whole-plasmid PCR (39) in pET44-TlyA, and expressed and purified by $\mathrm{Ni}^{2+}$-affinity chromatography as described above for wildtype TlyA. Protein folding and quality control was accomplished using nDSF on a Tycho NT.6 (NanoTemper) which monitors protein thermal unfolding using intrinsic fluorescence at 330 and 350 $\mathrm{nm}$. The unfolding profile and inflection temperature $\left(T_{i}\right)$ of each TlyA variant was determined using the instrument software for comparison to that of wild-type TlyA.

Isolation of Msm 50 S subunits - Msm 50 S subunits with unmethylated C2144 were isolated from a strain lacking TlyA activity (LR222 C101A) using following established procedures $(19,40)$. A small culture of Middlebrook 7H9 liquid medium was inoculated with a single colony of Msm LR222 C101A and grown overnight at $37^{\circ} \mathrm{C}$ with shaking (100 rpm). Fresh Middlebrook 7H9 medium (0.5-2 L) was inoculated with the overnight culture $\left(1 / 100\right.$ dilution) and the cultures grown for 72 hours at $37^{\circ} \mathrm{C}$ with shaking (100 rpm). Cells were harvested by low speed centrifugation for 10 minutes and washed (500 $\mathrm{mL}$ per $\mathrm{L}$ culture) twice with a solution of $10 \mathrm{mM} \mathrm{HEPES} / \mathrm{KOH}(\mathrm{pH} 7.6), 10 \mathrm{mM} \mathrm{MgCl}_{2}, 1 \mathrm{M} \mathrm{NH}_{4} \mathrm{Cl}$, and $6 \mathrm{mM} 2$-mercaptoethanol, and once with the same solution but with only $0.1 \mathrm{M} \mathrm{NH}_{4} \mathrm{Cl}$. The cells were then resuspended in the same final buffer and lysed using three passages through a French Press. After DNAse I addition, the lysate was cleared by centrifuging for 10 and 30 minutes (at 17,300 and $26,900 \times g$, respectively), and the resulting supernatant centrifuged at high speed for 18 hours $(277,200$ $\mathrm{x} g$ ) to pellet ribosomes. The 70S pellet was resuspended and dialyzed against a solution containing 10 $\mathrm{mM} \mathrm{HEPES} / \mathrm{KOH}$ ( $\mathrm{pH} 7.6$ ), $0.3 \mathrm{mM} \mathrm{MgCl}_{2}, 100 \mathrm{mM} \mathrm{NH}_{4} \mathrm{Cl}$, and $6 \mathrm{mM}$ 2-mercaptoethanol to split the ribosome subunits. $30 \mathrm{~S}$ and $50 \mathrm{~S}$ were then separated by centrifugation $(90,200 \times \mathrm{g})$ on a $10-30 \%$ sucrose gradient for 18 hours at $4{ }^{\circ} \mathrm{C}$. The resulting gradient was fractionated using an ÄKTA Purifier 
10 system to collect isolated $50 \mathrm{~S}$ subunits. Subunits were stabilized by addition of $\mathrm{MgCl}_{2}$ to $10 \mathrm{mM}$ and the solution centrifuged $(300,750 \times g)$ for 18 hours. The resulting $50 S$ subunit pellet was resuspended in a solution of $10 \mathrm{mM}$ HEPES/KOH (pH 7.6), $10 \mathrm{mM} \mathrm{MgCl}_{2}, 100 \mathrm{mM} \mathrm{NH}_{4} \mathrm{Cl}$, and $6 \mathrm{mM}$ 2mercaptoethanol, flash frozen, and stored at $-80^{\circ} \mathrm{C}$.

Cryo-EM sample preparation, data collection and structure determination-SAM analog NM6 (5'(diaminobutyric acid)-N-iodoethyl-5'-deoxyadenosine ammoniumhydrochloride) was prepared essentially as previously described (22) and purified by semi-preparative reverse-phase HPLC. A 3.0 $\mu \mathrm{L}$ mixture of purified Mtb TlyA, Msm 50S, and NM6 (at $0.5 \mu \mathrm{M}, 5 \mu \mathrm{M}$, and $10 \mu \mathrm{M}$ respectively) was applied to glow-discharged Quantifoil Cu R1.2/1.3 300 mesh grids. Grids were blotted at room temperature for 3.0-3.3 s at $>90 \%$ humidity and frozen in liquid ethane using a CP3 plunger. Cryo-EM data (3364 micrographs) were recorded as movies with defocus range of -0.8 to $-2.2 \mu \mathrm{m}$ at $81,000 \mathrm{x}$ magnification (1.0691 Å/pixel) on a Titan Krios 300 kV (TEM) with Gatan K3 direct electron detector at the National Center for CryoEM Access and Training (NCCAT). The dose per frame was 1.25 e/Å/frame (total dose of $50.79 \mathrm{e}^{-/} / \AA^{2}$ ) over a total exposure of $2 \mathrm{~s}$ divided over 40 frames (50 ms per frame).

Image alignment and dose-weighting were performed using Motioncor2 (41) and RELION3.0/3.1 (42) was used for subsequent data processing. The contrast transfer function was estimated using the program Gctf (43). To guide automatic picking, 1094 particles were manually picked and then classified into 2D classes. Automatic picking then selected 1,016,454 particles which were extracted with a box size of $280 \AA$. Multiple rounds of $2 \mathrm{D}$ classifications were performed to remove non-ribosomal particles before 3D refinement using a $60 \AA$ low-pass filtered reference map of the E. coli 50S (EMD3133). Iterative rounds of CTF refinement, 3D refinement, and 3D classification were performed resulting in a $3.05 \AA$ post-processed map (Fig. S2, center).

Prior to the final post-processing of the complete 50S-TlyA map, multibody refinement was also performed on the remaining particles with separate masks corresponding to TlyA/H69 and the remainder of the $50 \mathrm{~S}$, resulting in $3.89 \AA$ and $3.02 \AA$ resolution maps, respectively (Fig. S2, right). The final three maps (complete 50S-TlyA, TlyA/H69, and 50S alone) were then post-processed using Relion resulting in final $3.05 \AA, 3.61 \AA$, and $2.99 \AA$ maps, respectively, based on gold-standard refinement Fourier Shell Correlation ( 0.143 cutoff) (Fig. S2, S3). Local resolution maps were also generated using ResMap 1.1.4 (44).

All three final maps were used for model building and refinement. The $50 \mathrm{~S}$ model was created by docking an existing $M$. smegmatis $50 \mathrm{~S}$ structure (PDB code 5060), after de novo modeling of the NM6-modified C2144, into the 50S-TlyA map and using Coot (version 0.9-pre EL, ccpem) (24) and Phenix (version 1.19.2-4158-000) $(45,46)$. The TlyA model was generated using a TlyA CTD crystal structure (PDB code 5KYG) appended with a homology modeled NTD $(19,20)$. As initial docking of our 
hybrid model (19) did not give a satisfactory fit of the NTD into its portion of the map, this $\sim 60$ residue domain was manually re-built. The resulting complete full-length TlyA structure was then used as a search query in the Dali Protein Structure Comparison server (47). This search returned the unpublished structure of a putative hemolysin from Streptococcus thermophilus (PDB code 3HP7) as the closest structural homolog which was used to guide further improvement of our TlyA NTD model in regions of the map that were less well resolved. The model was subsequently split into separate TlyAH69 and remaining $50 S$ models and each separately real-space refined in Phenix $(48,49)$ using their respective multibody maps (using rigid-body and then to non-rigid body refinement). Finally, the refined models were recombined (without refinement) to create a final complete model of the 50S-TlyA complex and validated using Phenix $(45,46)$. Complete parameters for data collection and processing, and model building, refinement and validation are summarized in Table 1.

RT analysis of 23S rRNA methylation-Extent of methylation of the 50S subunit by TlyA WT and mutants was determined using a RT assay. Wild-type or variant Mtb TlyA (66 pmol, $2 \mu \mathrm{M})$ was incubated for 20 minutes at $37^{\circ} \mathrm{C}$ with Msm $50 \mathrm{~S}$ subunit $(33 \mathrm{pmol}, 1 \mu \mathrm{M})$ in the presence of SAM $(2.1$ $\mu \mathrm{M}$ ) in $10 \mathrm{mM}$ HEPES-KOH (pH 7.5), $10 \mathrm{mM} \mathrm{MgCl}_{2}, 50 \mathrm{mM} \mathrm{NH}_{4} \mathrm{Cl}$, and $5 \mathrm{mM}$ 2-mercaptoethanol. The reaction was terminated by phenol/chloroform extraction and the modified rRNA collected by ethanol precipitation. The rRNA modification at C2144 was assessed using an RT primer-extension reaction with a ${ }^{32} \mathrm{P}$-labeled DNA primer complementary to $23 \mathrm{~S}$ nucleotides 2188-2204. Modification of the 2'-O was observed only under conditions of complementary (dGTP) depletion (i.e. reactions with $75 \mu \mathrm{M}$ dATP, dUTP, dCTP; and $0.5 \mu \mathrm{M}$ dGTP). Controls with no TlyA or no dGTP depletion (i.e. $75 \mu \mathrm{M}$ dGTP) showed no RT stops corresponding to $\mathrm{C} 2144$ ribose modification. Extension products were run on a denaturing ( $50 \%$ urea) 8.6\% PAGE sequencing-style gel for 2 hours at $55 \mathrm{~W}$ and $50{ }^{\circ} \mathrm{C}$. Gels were dried and then imaged using a phosphor storage screen and Typhoon Trio Variable Mode Imager System (GE Healthcare). Extent of modification was estimated by band intensity comparison using ImageQuant TL 1D Version 7.0.

Methyltransferase activity assays using $\left.{ }^{3} \mathrm{H}\right]-\mathrm{SAM}-\mathrm{Quantitative} \mathrm{extent} \mathrm{of} \mathrm{methylation} \mathrm{of} \mathrm{the} 50 \mathrm{~S}$ subunit by wild-type and variant TlyA was determined using a filter-based enzyme assay with ${ }^{3} \mathrm{H}$-SAM. To establish optimal conditions for comparison to variant proteins, a time-course experiment was performed with wild-type TlyA. TlyA (final concentration $0.76 \mu \mathrm{M}$ ), Msm $50 \mathrm{~S}$ subunits (final concentration $0.38 \mu \mathrm{M}$ ), and ${ }^{3} \mathrm{H}$-SAM (final concentration $0.8 \mu \mathrm{M}$ ) were added to "Buffer $\mathrm{G}$ " (5 mM HEPES-KOH (pH 7.5), $50 \mathrm{mM} \mathrm{KCl,} 10 \mathrm{mM} \mathrm{NH}_{4} \mathrm{Cl}, 10 \mathrm{mM} \mathrm{MgOAc}$, and $6 \mathrm{mM}$ 2-mercaptoethanol) to a total reaction volume of $90 \mu \mathrm{L}$. The reaction was incubated for 60 minutes at $37{ }^{\circ} \mathrm{C}$, with $10 \mu \mathrm{L}$ aliquots (3.8 pmol 50S) removed and quenched in $140 \mu \mathrm{L} \%$ trichloroacetic acid at 0, 1, 2, 5, 10, 20, 40, and 60 minutes. The reaction was then applied to a glass microfiber filter and $50 \mathrm{~S}$ methylation quantified 
using scintillation counting of ${ }^{3} \mathrm{H}$ retained on the filter. A 20-minute time point was subsequently selected for comparison of wild-type and variant TlyA proteins using the assay performed essentially otherwise as described above.

Phylogenetic analysis of the TlyA protein family and residue conservation-TlyA homologs were retrieved from InterPro (IPR004538) with conserved sequence feature annotated for Hemolysin A/ rRNA methyltransferase TlyA family. Sequence redundancy was reduced in UniProt using the precalculated UniRef sequence clusters with a cutoff of $50 \%$ sequence identity. A total of 223 representative sequences were aligned using Clustal Omega and an unrooted neighbor joining phylogenetic tree was constructed using MEGA X (50) with evolutionary distances computed using the JTT matrix-based method (51). The rate variation among sites was modeled with a gamma distribution (shape parameter $=1$ ) and the residue propensities were calculated using Geneious.

\section{Acknowledgements}

We thank Dr. James Posey and colleagues at the Centers for Disease Control and Prevention, Atlanta GA for providing M. smegmatis strain LR222 C101A, and Drs. Puneet Juneja and Ricardo Guerrero for assistance with EM data collection and processing. This work was supported by the National Institutes of Health awards R01-AI088025 (GLC and CMD), T32-Al106699 (ZTL and PS), and T32-GM008602 (PS), and the Burroughs Wellcome Fund Investigator in the Pathogenesis of Infectious Disease award (CMD). This study was supported by the Robert P. Apkarian Integrated Electron Microscopy Core (IEMC) at Emory University, which is subsidized by the Emory School of Medicine and Emory College of Arts and Sciences. Some of this work was performed at the National Center for CryoEM Access and Training (NCCAT) and the Simons Electron Microscopy Center located at the New York Structural Biology Center, supported by the NIH Common Fund Transformative High Resolution Cryo-Electron Microscopy program (U24 GM129539), and by grants from the Simons Foundation (SF349247) and NY State Assembly.

\section{References}

1. J. Poehlsgaard, S. Douthwaite, The bacterial ribosome as a target for antibiotics. Nat Rev Microbiol 3, 870-881 (2005).

2. D. N. Wilson, Ribosome-targeting antibiotics and mechanisms of bacterial resistance. Nat Rev Microbiol 12, 35-48 (2014).

3. K. S. Long, B. Vester, "Resistance to antibiotics in bacteria through modification of nucleosides in 23S ribosomal RNA" in DNA and RNA modification enzymes: structure, mechanism, function and evolution, H. Grosjean, Ed. (Landes Bioscience, Austin, Tex., 2009), pp. 537-549. 
4. C. Fyfe, T. H. Grossman, K. Kerstein, J. Sutcliffe, Resistance to Macrolide Antibiotics in Public Health Pathogens. Cold Spring Harb Perspect Med 6 (2016).

5. G. L. Conn, M. Savic, R. Macmaster, "Resistance to antibiotics in bacteria through modification of nucleosides in 16S ribosomal RNA" in DNA and RNA modification enzymes: structure, mechanism, function and evolution, H. Grosjean, Ed. (Landes Bioscience, Austin, Tex., 2009), pp. 524-536.

6. Y. Doi, J. I. Wachino, Y. Arakawa, Aminoglycoside resistance: The emergence of acquired 16S ribosomal RNA methyltransferases. Infect Dis Clin North Am 30, 523-537 (2016).

7. D. M. Mikheil, D. C. Shippy, N. M. Eakley, O. E. Okwumabua, A. A. Fadl, Deletion of gene encoding methyltransferase (gidB) confers high-level antimicrobial resistance in Salmonella. J Antibiot (Tokyo) 65, 185-192 (2012).

8. J. Perdigao et al., GidB mutation as a phylogenetic marker for Q1 cluster Mycobacterium tuberculosis isolates and intermediate-level streptomycin resistance determinant in Lisbon, Portugal. Clin Microbiol Infect 20, O278-284 (2014).

9. C. E. Maus, B. B. Plikaytis, T. M. Shinnick, Mutation of tlyA confers capreomycin resistance in Mycobacterium tuberculosis. Antimicrob Agents Chemother 49, 571-577 (2005).

10. World Health Organization (2018). Global tuberculosis report 2018. https://apps.who.int/iris/handle/10665/274453

11. R. E. Stanley, G. Blaha, R. L. Grodzicki, M. D. Strickler, T. A. Steitz, The structures of the antituberculosis antibiotics viomycin and capreomycin bound to the $70 \mathrm{~S}$ ribosome. Nat Struct Mol Biol 17, 289-293 (2010).

12. L. Zhang et al., The structural basis for inhibition of ribosomal translocation by viomycin. Proc Natl Acad Sci U S A 117, 10271-10277 (2020).

13. Y. Lin et al., The antituberculosis antibiotic capreomycin inhibits protein synthesis by disrupting interaction between ribosomal proteins L12 and L10. Antimicrob Agents Chemother 58, 2038-2044 (2014).

14. K. Yang et al., Structural insights into species-specific features of the ribosome from the human pathogen Mycobacterium tuberculosis. Nucleic Acids Res 45, 10884-10894 (2017).

15. T. Monshupanee, S. K. Johansen, A. E. Dahlberg, S. Douthwaite, Capreomycin susceptibility is increased by TlyA-directed 2'-O-methylation on both ribosomal subunits. Mol Microbiol 85, 11941203 (2012).

16. S. K. Johansen, C. E. Maus, B. B. Plikaytis, S. Douthwaite, Capreomycin binds across the ribosomal subunit interface using tlyA-encoded 2 '-O-methylations in $16 \mathrm{~S}$ and $23 \mathrm{~S}$ rRNAs. Mol Cell 
23, 173-182 (2006).

17. A. Salamaszynska-Guz, B. Taciak, A. Kwiatek, D. Klimuszko, The Cj0588 protein is a Campylobacter jejuni RNA methyltransferase. Biochem Bioph Res Co 448, 298-302 (2014).

18. P. Freihofer et al., Nonmutational compensation of the fitness cost of antibiotic resistance in mycobacteria by overexpression of tlyA rRNA methylase. RNA 22, 1836-1843 (2016).

19. M. A. Witek, E. G. Kuiper, E. Minten, E. K. Crispell, G. L. Conn, A Novel Motif for S-Adenosyl-Imethionine Binding by the Ribosomal RNA Methyltransferase TlyA from Mycobacterium tuberculosis. J Biol Chem 292, 1977-1987 (2017).

20. N. E. Arenas et al., Molecular modeling and in silico characterization of Mycobacterium tuberculosis TlyA: possible misannotation of this tubercle bacilli-hemolysin. BMC Struct Biol 11, 16 (2011).

21. V. Stanevich et al., The structural basis for tight control of PP2A methylation and function by LCMT1. Mol Cell 41, 331-342 (2011).

22. R. L. Weller, S. R. Rajski, Design, synthesis, and preliminary biological evaluation of a DNA methyltransferase-directed alkylating agent. Chembiochem 7, 243-245 (2006).

23. J. Hentschel et al., The complete structure of the Mycobacterium smegmatis $70 \mathrm{~S}$ ribosome. Cell Rep 20, 149-160 (2017).

24. P. Emsley, K. Cowtan, Coot: model-building tools for molecular graphics. Acta Crystallogr D Biol Crystallogr 60, 2126-2132 (2004).

25. P. V. G. Sergiev, A. Y.; Prokhorova, I. V.; Sergeeva, O. V.; Osterman, I. A.; Nesterchuk, M. V., "Modifications of ribosomal RNA: from enzymes to function" in Ribosomes: Structure, function, and dynamics, M. V. W. Rodnina, W.; Green, R. , Ed. (Springer-Verlag/Wien, Vienna, 2011), pp. 97110.

26. I. A. Osterman, O. A. Dontsova, P. V. Sergiev, rRNA Methylation and Antibiotic Resistance. Biochemistry (Mosc) 85, 1335-1349 (2020).

27. H. L. Schubert, R. M. Blumenthal, X. Cheng, Many paths to methyltransfer: a chronicle of convergence. Trends Biochem Sci 28, 329-335 (2003).

28. C. Davies, R. B. Gerstner, D. E. Draper, V. Ramakrishnan, S. W. White, The crystal structure of ribosomal protein S4 reveals a two-domain molecule with an extensive RNA-binding surface: one domain shows structural homology to the ETS DNA-binding motif. EMBO J 17, 4545-4558 (1998).

29. J. A. Dunkle et al., Molecular recognition and modification of the $30 \mathrm{~S}$ ribosome by the aminoglycoside-resistance methyltransferase NpmA. Proc Natl Acad Sci U S A 111, 6275-6280 (2014). 
30. N. Zelinskaya, M. A. Witek, G. L. Conn, The pathogen-derived aminoglycoside resistance $16 S$ rRNA methyltransferase NpmA possesses dual m1A1408/m1G1408 specificity. Antimicrob Agents Chemother 59, 7862-7865 (2015).

31. K. Vinal, G. L. Conn, Substrate recognition and modification by a pathogen-associated aminoglycoside resistance 16S rRNA methyltransferase. Antimicrob Agents Chemother 61 (2017).

32. N. Huang, N. K. Banavali, A. D. MacKerell, Jr., Protein-facilitated base flipping in DNA by cytosine5-methyltransferase. Proc Natl Acad Sci U S A 100, 68-73 (2003).

33. M. Nosrati et al., Functionally critical residues in the aminoglycoside resistance-associated methyltransferase RmtC play distinct roles in 30 S substrate recognition. J Biol Chem 294, 1764217653 (2019).

34. J. E. Phelan et al., Integrating informatics tools and portable sequencing technology for rapid detection of resistance to anti-tuberculous drugs. Genome Med 11, 41 (2019).

35. I. B. Olawoye et al., Whole genome sequencing of clinical samples reveals extensively drug resistant tuberculosis (XDR TB) strains from the Beijing lineage in Nigeria, West Africa. Sci Rep 11, 17387 (2021).

36. Q. Li et al., Mutation and transmission profiles of second-line drug resistance in clinical isolates of drug-resistant Mycobacterium tuberculosis from Hebei Province, China. Front Microbio/ 10, 1838 (2019).

37. T. M. Walker et al., A cluster of multidrug-resistant Mycobacterium tuberculosis among patients arriving in Europe from the Horn of Africa: a molecular epidemiological study. Lancet Infect Dis 18, 431-440 (2018).

38. J. Zhao et al., Assessing capreomycin resistance on tlyA deficient and point mutation (G695A) Mycobacterium tuberculosis strains using multi-omics analysis. Int J Med Microbiol 309, 151323 (2019).

39. K. Miyazaki, MEGAWHOP cloning: a method of creating random mutagenesis libraries via megaprimer PCR of whole plasmids. Methods Enzymol 498, 399-406 (2011).

40. A. K. Singh, J. M. Reyrat, Laboratory maintenance of Mycobacterium smegmatis. Curr Protoc Microbiol Chapter 10, Unit10C.11 (2009).

41. X. Li et al., Electron counting and beam-induced motion correction enable near-atomic-resolution single-particle cryo-EM. Nat Methods 10, 584-590 (2013).

42. S. H. Scheres, RELION: implementation of a Bayesian approach to cryo-EM structure determination. J Struct Biol 180, 519-530 (2012). 
43. K. Zhang, Gctf: Real-time CTF determination and correction. J Struct Biol 193, 1-12 (2016).

44. A. Kucukelbir, F. J. Sigworth, H. D. Tagare, Quantifying the local resolution of cryo-EM density maps. Nat Methods 11, 63-65 (2014).

45. P. V. Afonine et al., New tools for the analysis and validation of cryo-EM maps and atomic models. Acta Crystallogr D Struct Biol 74, 814-840 (2018).

46. C. J. Williams et al., MolProbity: More and better reference data for improved all-atom structure validation. Protein Sci 27, 293-315 (2018).

47. L. Holm, DALI and the persistence of protein shape. Protein Sci 29, 128-140 (2020).

48. P. V. Afonine et al., Real-space refinement in PHENIX for cryo-EM and crystallography. Acta Crystallogr D Struct Biol 74, 531-544 (2018).

49. D. Liebschner et al., Macromolecular structure determination using X-rays, neutrons and electrons: recent developments in Phenix. Acta Crystallogr D Struct Biol 75, 861-877 (2019).

50. S. Kumar, G. Stecher, M. Li, C. Knyaz, K. Tamura, MEGA X: Molecular evolutionary genetics analysis across computing platforms. Mol Biol Evol 35, 1547-1549 (2018).

51. D. T. Jones, W. R. Taylor, J. M. Thornton, The rapid generation of mutation data matrices from protein sequences. Comput Appl Biosci 8, 275-282 (1992). 


\section{Tables}

Table 1. Cryo-EM data collection, refinement and
model validation for the 50 S-TlyA complex.

\begin{tabular}{|c|c|}
\hline $\begin{array}{l}\text { Deposition } \\
\text { Coordinates (RCSB PDB) } \\
\text { Map (EMDB) }\end{array}$ & $\begin{array}{c}7 S 0 S \\
\text { EMD-24792 }\end{array}$ \\
\hline $\begin{array}{l}\text { Data Collection/ Processing } \\
\text { Microscope } \\
\text { Camera } \\
\text { Voltage, kV } \\
\text { Magnification } \\
\text { Electron exposure, } \mathrm{e}^{-} / \AA^{2} \\
\text { Defocus range } \\
\text { Pixel size, } \AA \\
\text { Symmetry } \\
\text { No. particles } \\
\text { Initial } \\
\text { Final } \\
\text { Map resolution (FSC 0.143), } \AA\end{array}$ & $\begin{array}{c}\text { TFS Titan Krios } \\
\text { Gatan K3 } \\
300 \\
81,000 \mathrm{x} \\
50.79 \\
-0.8 \text { to }-2.2 \\
1.069 \\
\text { C1 }\end{array}$ \\
\hline $\begin{array}{l}\text { Refinement and Model } \\
\text { Model resolution (FSC 0.143), } \AA \\
\text { CC } \\
\text { Map sharpening, } \AA^{2} \\
\text { 50S-TlyA } \\
\text { TlyA-H69 (multibody) } \\
\text { 50S alone (multibody) }\end{array}$ & $\begin{array}{c}3.0 \\
0.66 \\
\\
83.1 \\
109.5 \\
72.9\end{array}$ \\
\hline $\begin{array}{l}\text { Non-hydrogen atoms } \\
\text { Protein residues } \\
\text { RNA residues } \\
\text { Ligand/ modified nucleotide }\end{array}$ & $\begin{array}{c}3,946 \\
3,236 \\
407\end{array}$ \\
\hline $\begin{array}{l}\text { B factors (min/max/mean), } \AA^{2} \\
\text { Protein } \\
\text { RNA } \\
\text { Ligand/ modified nucleotide } \\
\text { RMS deviations } \\
\text { Bond lengths, } \AA \\
\text { Bond angles, }\end{array}$ & $\begin{array}{l}0.21 / 172.4 / 17.1 \\
0.05 / 190.2 / 19.7 \\
0.78 / 109.5 / 17.3\end{array}$ \\
\hline $\begin{array}{l}\text { Validation } \\
\text { MolProbity score } \\
\text { Clashscore } \\
\text { Poor rotamers, \% } \\
\text { Protein }\end{array}$ & $\begin{array}{l}1.99 \\
9.68 \\
1.21\end{array}$ \\
\hline $\begin{array}{l}\text { Ramachandran plot } \\
\text { Favored, \% } \\
\text { Allowed, \% } \\
\text { Disallowed, \% }\end{array}$ & $\begin{array}{l}93.79 \% \\
6.21 \% \\
0 \%\end{array}$ \\
\hline $\begin{array}{l}\text { RNA } \\
\text { Pucker outliers, \% } \\
\text { Bond outliers, \% } \\
\text { Angle outliers, \% }\end{array}$ & $\begin{array}{l}0 \% \\
0 \% \\
0.1 \%\end{array}$ \\
\hline
\end{tabular}




\section{Figure Legends}

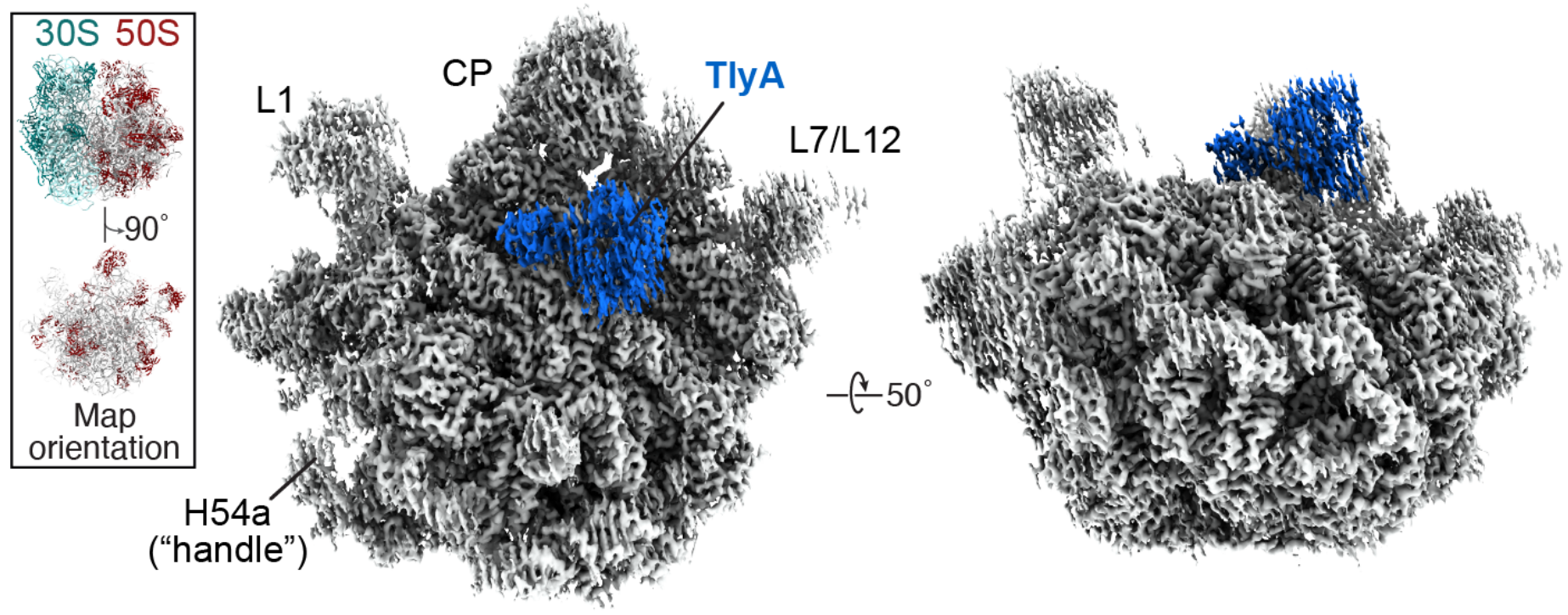

Figure 1. Cryo-EM map at 3.05 A resolution of the 50S-TlyA complex. Two views of the cryo-EM map for the 50S:TlyA complex. The inset (left) shows a cartoon of the 70S Msm ribosome and rotated $50 S$ subunit to indicate the view of the left map (toward the subunit interface). TlyA (blue) is bound to the $50 \mathrm{~S}$ subunit (white) on the subunit interface over $\mathrm{H} 69$ containing the modification site (residue C2144). Key 50 S features are indicated: L1 stalk (L1), central protuberance (CB), the L7/L12 stalk (L7/L12) and H54a (also known and the handle). H54a, which extends outward and interacts extensively with $30 \mathrm{~S}$ in the intact $70 \mathrm{~S}$ ribosome, was partially observed on the $50 \mathrm{~S}$ surface in proximity to TlyA, but with weaker map features close to the enzyme. 


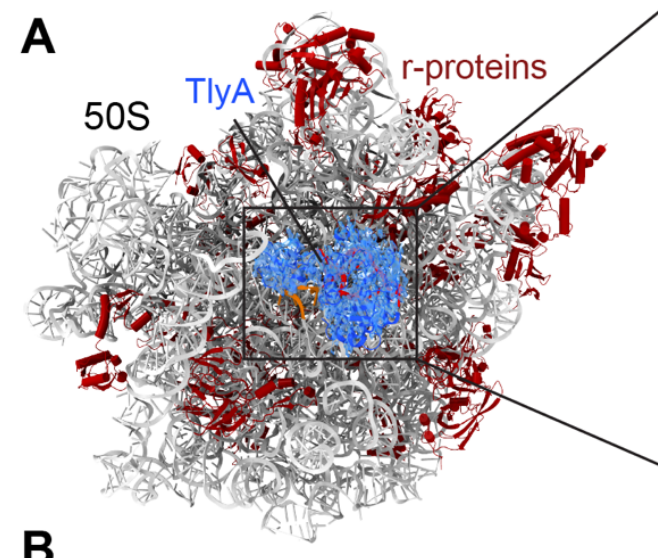

B

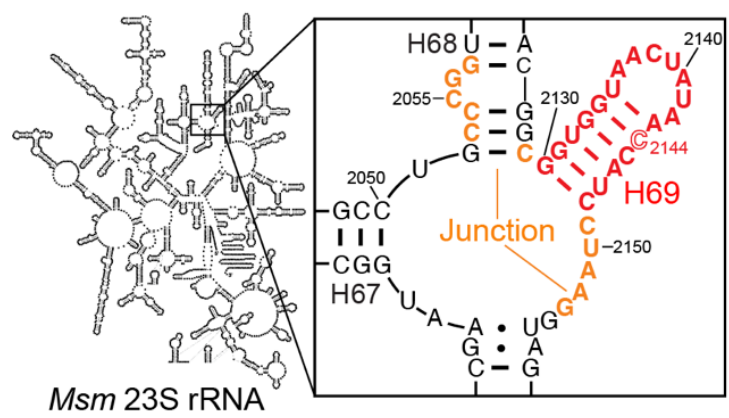

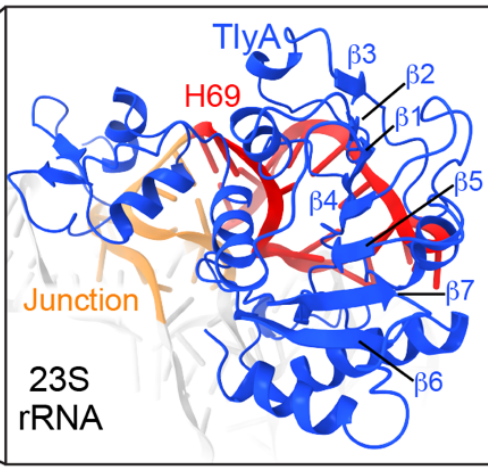

D

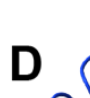

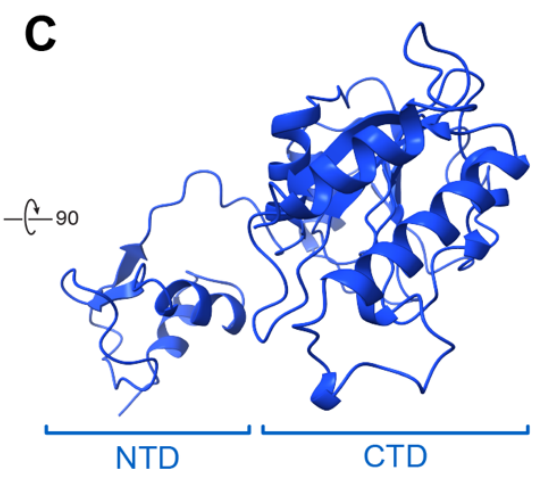

E
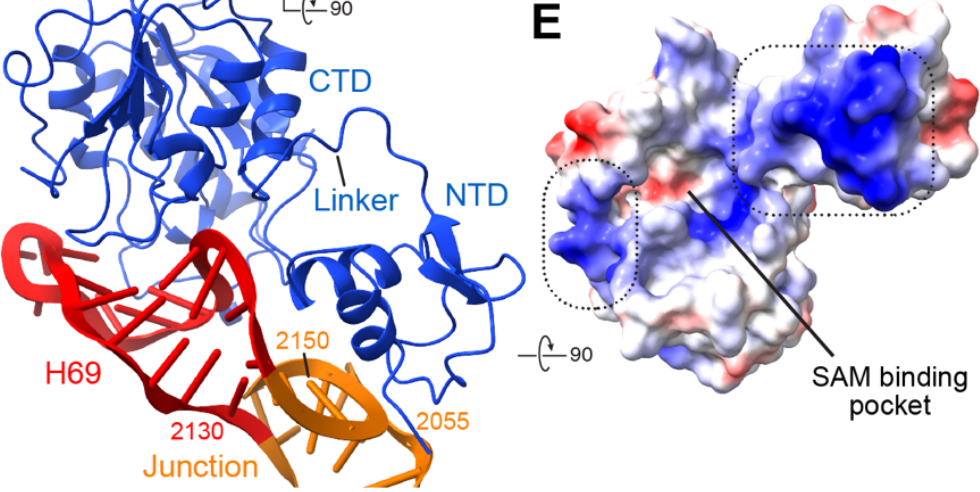

Figure 2. TlyA binds to 23S rRNA H69 and the adjacent rRNA junction via surface of positively

charged residues. $A$, Structure of the 50S-TlyA complex with TlyA (blue cartoon, with semitransparent surface) bound at $\mathrm{H} 69$ on the subunit interface. Ribosomal proteins are shown in dark red and 23S rRNA in white. Inset, zoomed-in view of TlyA (blue) bound to H69 (red) and the adjacent rRNA junction (junction; orange). $B, M$. smegmatis $23 S$ rRNA secondary structure, highlighting the sequence of regions bound by TlyA, H69 (red) and junction (orange). The target nucleotide $\mathrm{C} 2144$ is shown in outline font. C, Modeled structure of full-length TlyA shown in an orthogonal view to panel $A$ and comprising an N-terminal ribosomal protein S4 fold (NTD) and a C-terminal Class I methyltransferase fold with a seven $\beta$-strand core ( $\beta 1-\beta 7$, labeled in panel $A)$ surrounded by $\alpha$-helices. $D$, The TlyAH69/junction interaction viewed from the 50S surface. The TlyA NTD binds at the base of H69 (red) and the adjacent 23S rRNA junction (orange), while the TlyA CTD interacts exclusively with H69 nucleotides around the modification site. E. Electrostatic surface of TlyA revealing two main patches of positivelycharged (blue, dashed boxes) surface along the face of TlyA interacting with the rRNA. The negativelycharged (red) cosubstrate-binding pocket is indicated between the two positively-charged patches. 


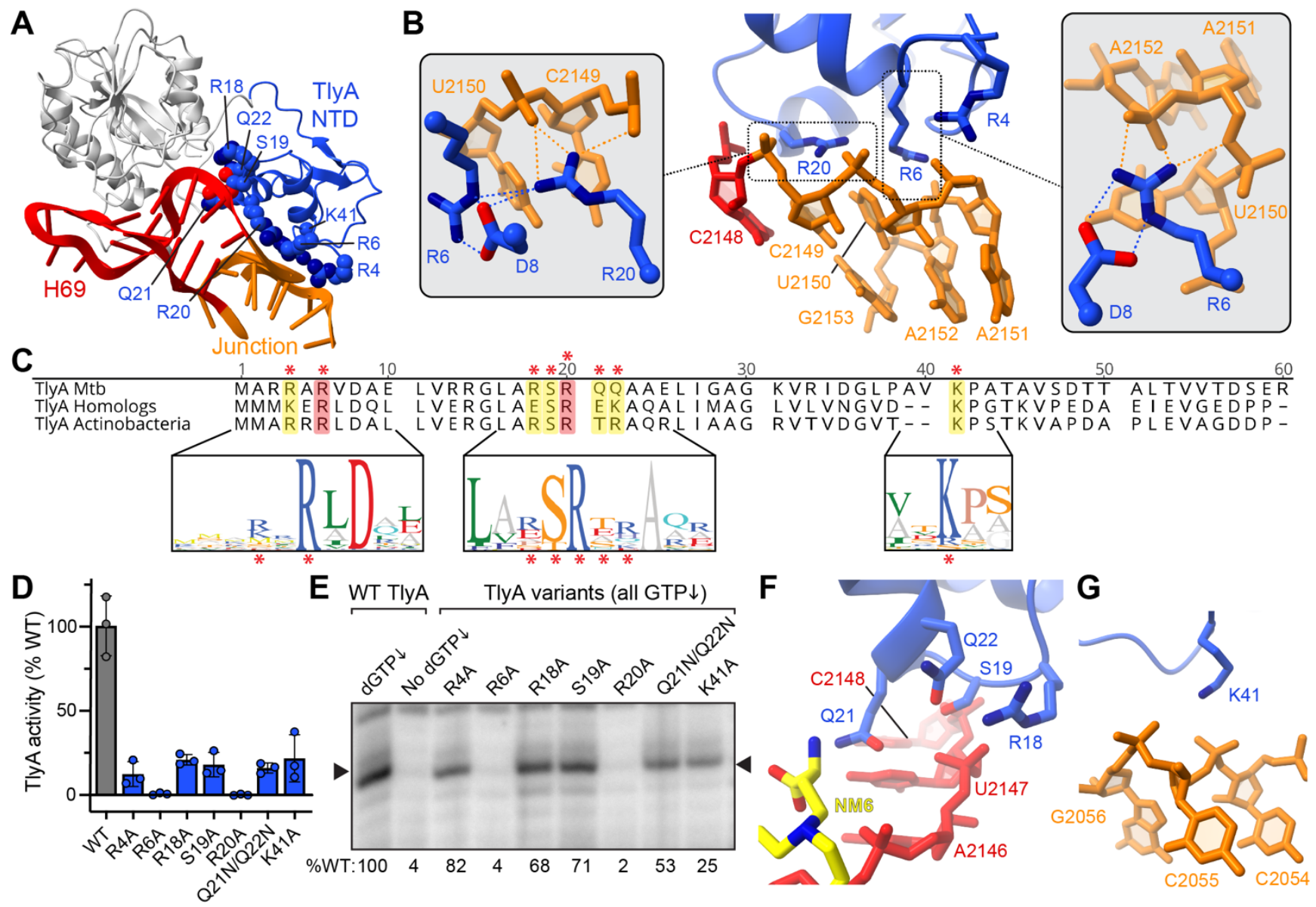

Figure 3. TlyA NTD recognizes a complex rRNA structure at the base of H69. A, Overview of the TlyA-H69 complex highlighting NTD residues on the TlyA interaction surface for which amino acid substitutions were made. $\boldsymbol{B}$, Zoomed in view of interactions made by TlyA NTD residues Arg4, Arg6 and Arg20 with nucleotides of the rRNA junction proximal to H69 (orange). Insets: alternate views of Arg6/Arg20 and Arg6 alone with interactions with rRNA and between protein residues indicated with orange and blue dotted lines, respectively. $\boldsymbol{C}$, Sequence alignment of the Mtb NTD sequence with the consensus sequences for all TlyA homologs and closer homologs from actinobacteria only. Shown below are sequence logo plot representations of sequence conservation for the selected regions among actinobacterial TlyA sequences. The red asterisk denotes sites of amino acid substitutions generated in this work. $\boldsymbol{D}$, In vitro methylation of Msm 50 S by wild-type (normalized to $100 \%$ ) and variant assay TlyA proteins using [ $\left.{ }^{3} \mathrm{H}-\mathrm{SAM}\right]$. $\boldsymbol{E}$, Representative gel showing the results of RT analysis of Msm 50 S methylation by variant assay TlyA proteins via RT using a radiolabeled DNA primer. Stops at methylated ribose are only observed under conditions of depleted dGTP (compare first two lanes with wild-type TlyA). Values below the image are the average band intensity relative to wild-type TlyA for at least two independent experiments. Zoomed views of the interactions made by TlyA NTD residues $\boldsymbol{F}$, Arg18, Ser19, GIn21 and GIn22, and G, Lys41. 


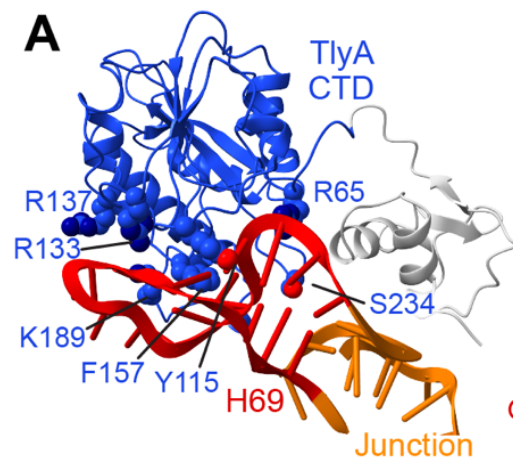

E
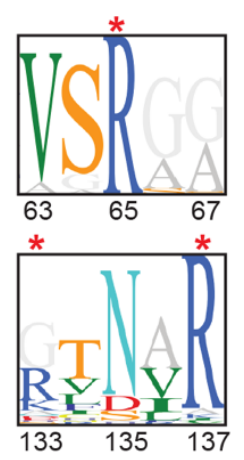

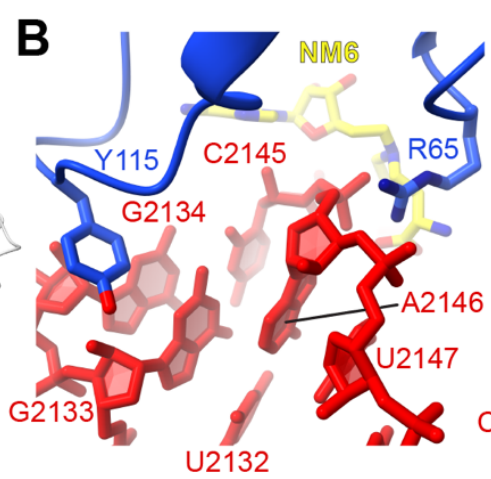

$\mathbf{F}$

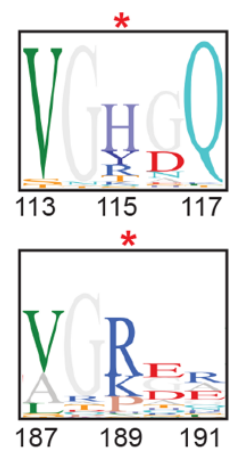

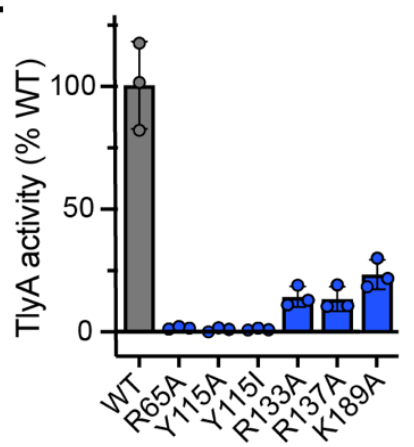

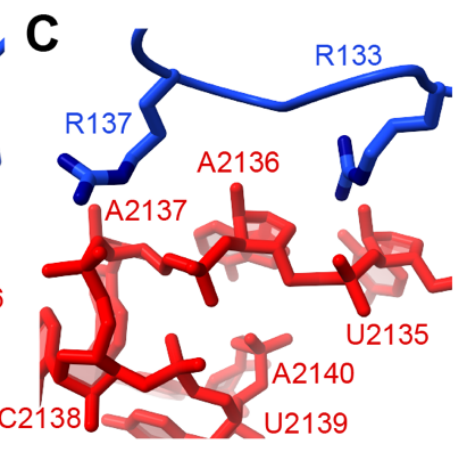

D

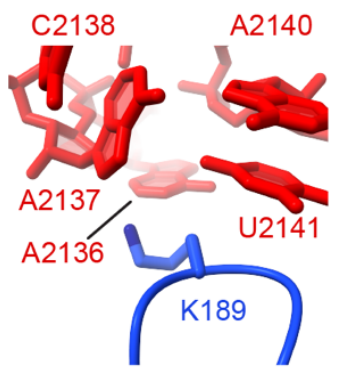

G WT TlyA, TlyA variants (all dGTP $\downarrow$ )

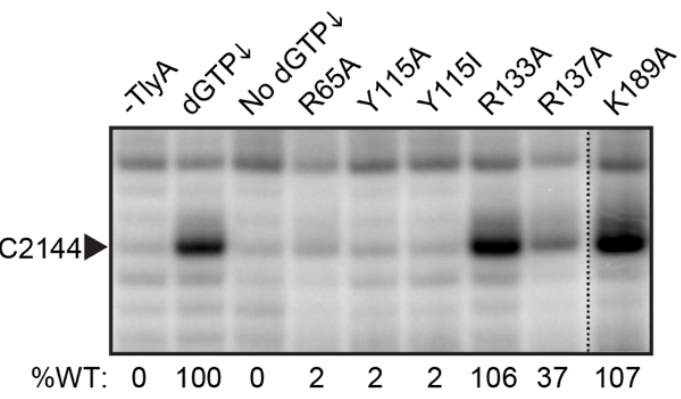

Figure 4. The TlyA CTD interacts with $\mathrm{H} 69$ surrounding the modification site. $A$, Overview of the

TlyA-H69 complex highlighting CTD residues on the TlyA interaction surface for which amino acid substitutions were made. Zoomed in views of interactions between $\mathrm{H69}$ (red) and TlyA CTD residues $\boldsymbol{B}$, Arg65, Tyr115, C, Arg133, Arg137, and D, Lys189. E, Sequence logo plot representations of actinobacterial TlyA sequence conservation for regions surrounding the selected CTD residues. The red asterisk denotes sites of amino acid substitutions made in this work. $F$, In vitro methylation of $\mathrm{Msm}$ $50 \mathrm{~S}$ using ${ }^{3} \mathrm{H}$-SAM for TlyA WT and CTD variant proteins. G, Representative gel showing the results of RT analysis of Msm 50 S methylation by TlyA CTD variants. Values below the image are the average band intensity relative to wild-type TlyA for at least two independent experiments. 

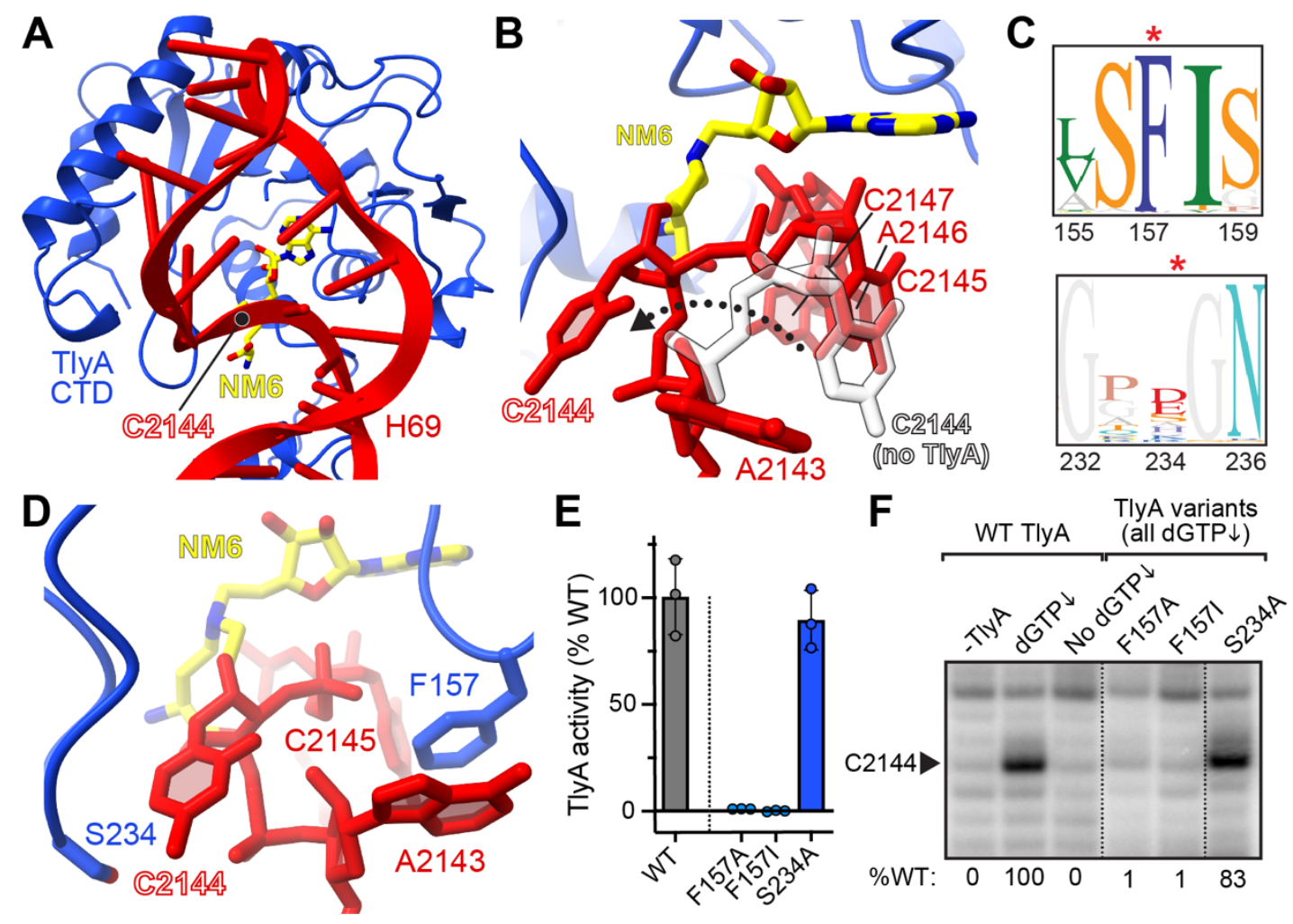

Figure 5. TlyA uses a base flipping mechanism to position C2144 for 2'-OH modification. A, View of TlyA CTD and NM6 cosubstrate positioned over the $\mathrm{H} 69$ modification site. $\boldsymbol{B}$, The NM6-modified C2144 nucleotide is flipped from $\mathrm{H} 69$ compared to its original position (white, semi-transparent sticks). $\boldsymbol{C}$, Sequence logo plot representations of actinobacterial TlyA sequence conservation for regions surrounding the selected CTD residues proximal to the flipped nucleotide. The red asterisk denotes sites of amino acid substitutions generated in this work. $\boldsymbol{D}$, TlyA CTD residues Phe157 and Ser234 interact with $\mathrm{H} 69$ nucleotide $\mathrm{C} 2144$ and A2143, respectively. $\boldsymbol{E}$, In vitro methylation of Msm $50 \mathrm{~S}$ using ${ }^{3} \mathrm{H}$-SAM for TlyA WT and indicated CTD variant proteins. $\boldsymbol{F}$, Representative gel showing the results of RT analysis of Msm 50 S methylation by these TlyA CTD variants. Values below the image are the average band intensity relative to wild-type TlyA for at least two independent experiments. In panels $E$ and F, data for wild-type TlyA are the same as in Fig. 4 (dotted lines denote regions removed from the original images). 